\title{
ChemComm
}

\section{Exceptional function of nanoporous metal organic framework particles in emulsion stabilisation $†$}

Cite this: Chem. Commun., 2013,

49, 8208

Received 16th May 2013,

Bo Xiao, ${ }^{\star a b}$ Qingchun Yuan ${ }^{c}$ and Richard A. Williams ${ }^{d}$

Accepted 17th July 2013

DOI: $10.1039 /$ c3cc43689f

www.rsc.org/chemcomm

A new concept of nanoporous metal organic framework particles stabilising emulsions was investigated. The copper benzenetricarboxylate MOF particles adsorbed at the oil/water interface play an exceptional role in stabilising both oil-in-water and water-in-oil emulsions.

Pickering emulsion is of importance in numerous industrial applications involving food, pharmaceutics, cosmetics, oil well drilling and fuel conversion. ${ }^{1}$ It is achieved by adsorption of small size solid particles at the interface of immiscible liquid phases. The nature of the solid particle is the key factor affecting the stabilisation of emulsions; other factors include the composition of the oil phase, salinity, $\mathrm{pH}$ and temperature. In order to stabilise emulsions effectively, the solid particles are required to have hydrophilic and hydrophobic characteristics similar to surfactants, thus can be wetted by both oil and aqueous phases with an ideal contact angle $\sim 90^{\circ}$ at the interface. ${ }^{2}$ Adsorption of the solid particles at the interface is normally irreversible. The desorption requires much higher energy than the thermal energy at ambient temperature. ${ }^{3}$ This differentiates solid particle stabilisers from surfactants used in emulsion stabilisation. A number of micro/nanoparticles, such as silica, bacteria, proteins, gold nanoparticles, clay, metal oxides, carbon black and latex colloids, have been used for stabilising emulsions. ${ }^{1 e, 4}$ For these particles to achieve the desired emulsions, the surface modification is the prerequisite. We herein report a new approach to use nanoporous metal organic framework (MOF) particles to stabilise emulsions.

Different from the other solids, nanoporous MOFs are a new class of crystalline materials, consisting of organic ligands and inorganic metal cations, which have a high surface area $\sim 1000-7000 \mathrm{~m}^{2} \mathrm{~g}^{-1}$. Their diverse coordination and organic functionalities give MOFs the

\footnotetext{
${ }^{a}$ School of Chemistry \& Chemical Engineering, Queen's University Belfast, David Keir Building, Stranmillis Road, Belfast BT9 5AG, Northern Ireland, UK. E-mail: b.xiao@qub.ac.uk

${ }^{b}$ Taiyuan University of Technology, Taiyuan, P. R. China

${ }^{c}$ Chemical Engineering and Applied Chemistry, School of Engineering and Applied Sciences, Aston University, Birmingham B4 7ET, UK

${ }^{d}$ The College of Engineering and Physical Sciences, Faculty of Engineering, The University of Birmingham, Birmingham B15 2TT, UK

$\dagger$ Electronic supplementary information (ESI) available: Experimental procedure and X-ray diffraction. See DOI: $10.1039 / \mathrm{c} 3 \mathrm{cc} 43689 \mathrm{f}$
}

tunable shape and size of nanopores and unique hydrophilic and hydrophobic features. The guest molecules adsorbed in the pores to some extent provide MOFs with an 'additional' functionality. These characteristics of MOFs enable wide potential applications, e.g. hydrogen storage, anticancer drug delivery, catalytic reaction and electrochemical applications. ${ }^{5}$ In making new microstructured objects through Pickering emulsion, MOFs will have distinct advantages over other solids currently in use. To the best of our knowledge, relevant studies on this topic have been rare until now.

In this study, the nanoporous MOF nanoparticles were prepared at ambient temperature using a simple stirring reaction method (see ESI $\dagger$ ), which could be more easily scaled up than a hydrothermal method. 1,3,5-Benzenetricarboxylic acid (H3BTC) reacted with copper acetate in an ethanol/water medium, yielding a turquoise blue coloured MOF slurry (Cu-BTC). The obtained Cu-BTC particles have an effective diameter $c a \sim 291 \mathrm{~nm}$ with a polydispersity of 0.113 and a zeta potential of $-0.3 \mathrm{mV}$. The X-ray diffraction patterns of Cu-BTC particles were in good agreement with those of single crystals produced by the hydrothermal method (Fig. S1, ESI $\dagger$ ).

The structure of nanoporous Cu-BTC MOFs (HKUST-1 ${ }^{6}$ ) is illustrated in Fig. S2 (ESI $\dagger$ ). The square-shaped pores account for around $40 \%$ of the material porosity, which are accessible to many interesting molecules such as hydrogen, bioactive nitric oxide, pyridine and 4 -(methylamino) pyridine. ${ }^{5 f, 7}$ Furthermore, the hybrid structure built by coordination of copper cations and benzenetricarboxylate endows the Cu-BTC particles with a combined hydrophilic and lipophilic feature that is necessary for solid particles to stabilise the water-in-oil or the oil-in-water dispersion system. The next concern was the feasibility of MOF particles in stabilising emulsions without further surface modification.

The aqueous dispersion of the Cu-BTC nanoparticles was used as the aqueous phase for the subsequent preparation of oil-water emulsions. In this study, the emulsion type was defined by a lower volume ratio of the disperse phase to the continuous phase favouring the formation of disperse droplets. Both types of emulsions (oilin-water and water-in-oil) were successfully prepared using a rotorstator high speed homogenization method (Fig. 1a and e). In the water-in-oil emulsion (Fig. 1a), the aqueous droplets were heavier than the oil and therefore creamed down to the bottom of the 


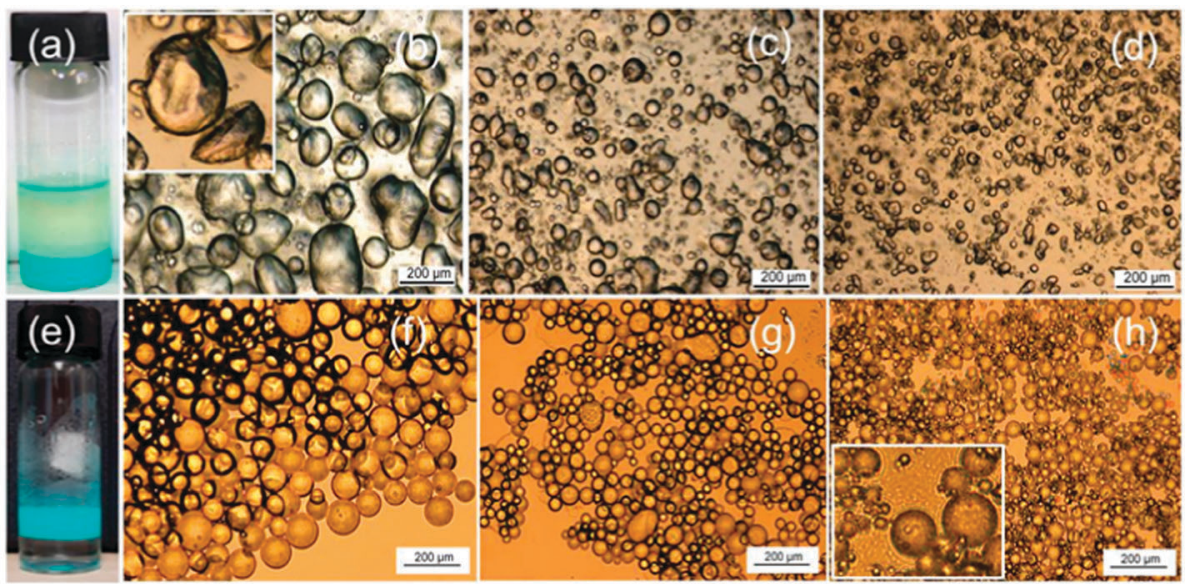

Fig. 1 Cu-BTC particles stabilise both water-in-oil and oil-in-water emulsions. Top: (a) water-in-oil emulsion (1.0 ml of aqueous suspension in $3.0 \mathrm{ml}$ of oil), with MOF particle concentrations: (b) $0.8 \mathrm{wt} \%$; (c) $1.6 \mathrm{wt} \%$ and (d) $3.2 \mathrm{wt} \%$. Lower: (e) oil-in-water emulsions; (f) $1.0 \mathrm{ml}$ of oil and $0.8 \mathrm{wt} \% \mathrm{MOFs}$; (g) $1.0 \mathrm{ml}$ of oil and $1.8 \mathrm{wt} \%$ MOFs; (h) $0.5 \mathrm{ml}$ of oil and $1.8 \mathrm{wt} \% \mathrm{MOFs}$, in $2.0 \mathrm{ml}$ of aqueous phase.

container. After a few hours, a translucent and colourless oil layer was formed at the top. In contrast, the oil droplets in the oil-in-water emulsion (Fig. 1e) were lighter than the aqueous phase and thus creamed up as the blue layer. Underneath was a clear aqueous phase without the precipitated particles at the bottom. It suggests all of the Cu-BTC nanoparticles being virtually adsorbed at the oil-water interface in a monolayer manner. These emulsions were stable in a sealed container without the droplet appearance changing.

The droplet appearance was studied by the examination of a single layer of the droplets using an optical microscope. The droplets in both types of emulsions clearly identified themselves from the continuous phase. The aqueous droplets dispersed in the oil gradually lost their inner volume, this was caused by the evaporation of the aqueous phase, and thus led the surface to become wrinkled with a clearly visible skin/shell as shown in the inset of Fig. 1b. This confirms that the MOF particles have been adsorbed at the oil-water interface to form a physical barrier preventing droplets from coalescing. Compared with water droplets, the oil droplets kept their spherical shape for a longer time due to the lower volatility of the oil. Coalescence under the action of capillary force was observed with the continuous phase disappearing. We also examined another type of MOF particles, e.g. the MOF CPO-27 (Co; Ni). ${ }^{5 h, i}$ The initial results showed the Co MOF performed well in stabilising both the oil-in-water and water-in-oil emulsions than the Ni MOF. Further studies are required to unveil the complex effects of MOF chemical composition and morphology.

The droplet sizes are mainly affected by two factors: the particle population in the emulsion system and the kinetics of solid particle adsorption at the oil-water interface. Reducing the droplet size will increase the interfacial area. A large particle population is therefore required in place to facilitate particles to be spontaneously adsorbed onto the fresh droplet surface to stabilise the droplets. Both the waterin-oil and the oil-in-water systems (Fig. 1) followed this general rule. Additionally, the oil viscosity and particle diffusion from the continuous phase to the interface were also observed to influence water and oil droplet sizes. With particle concentration increasing from 0.8 to $3.2 \mathrm{wt} \%$ (Fig. 1b-d), the size of water-in-oil droplets decreased from $\sim 150$ to $\sim 10 \mu \mathrm{m}$. Under rotor-stator homogenization conditions, a very strong turbulent shear force was applied for two minutes, which gave the droplet sufficient time to break and coalesce repeatedly until the droplet size and the particle adsorption reached quasiequilibrium.

Similar phenomena were observed in the oil-in-water system (Fig. 1f-h). However, the droplets in Fig. 1h show a high polydispersity. The emulsion system contained $0.5 \mathrm{ml}$ of the oil and $2.0 \mathrm{ml}$ of the aqueous phase with $1.8 \mathrm{wt} \% \mathrm{Cu}-\mathrm{BTC}$ particles, which had the highest ratio of the particle population to the disperse phase volume, $\sim 7.2$ (particle concentration $\times$ suspension volume) $/ 1.0 \mathrm{ml}$. Comparison of the results in Fig. $1 \mathrm{~g}$ and $\mathrm{h}$ showed that at a given particle concentration a lower oil ratio (Fig. $1 \mathrm{~h}$ ) tended to produce a large population of smaller droplets. They coexisted with the big droplets similar to that in Fig. 1g. This reflected the cooperative effect of the droplet breakage under the strong turbulent conditions and the particle dynamic adsorption at the freshly formed oil droplet surfaces. The formation of droplets followed the mechanism that the droplet breakage accompanied with particle stabilisation occurred in a series of stages. Only under a condition that there were sufficient free particles present in the system at each stage, stable uniform droplets could be formed.

In addition to particle diffusion, particle wetting kinetics also plays an important role in efficient homogenization, especially when the microfluidic/membrane emulsification method was used to produce droplets with precisely controlled sizes. ${ }^{8}$ Different from rotor-stator homogenisation, the membrane method undertakes a different droplet formation mechanism. The droplets were formed through the membrane pores in a drop-by-drop manner, as shown by a high speed camera image (Fig. 2a). In this process, if the stabiliser is effectively adsorbed onto the oil-water interface spontaneously, the formed droplets will proportionally respond to the pore size and uniformity. As a result, the droplet size and distribution could be maintained in the final product.

In the membrane emulsification, approximately $10 \mathrm{ml}$ of the oil was dispersed into $30 \mathrm{ml}$ of the aqueous phase that contained $1.8 \mathrm{wt} \%$ of the $\mathrm{Cu}-\mathrm{BTC}$ nanoparticles. In this case, the number of particles was in excess. The prepared sample was then separated into three layers after standing for a while (Fig. 2b), i.e. a top oil droplet layer stabilised by the Cu-BTC particles, a middle clear aqueous phase and a bottom blue layer with the precipitated free $\mathrm{Cu}-\mathrm{BTC}$ nanoparticles. The sizes 

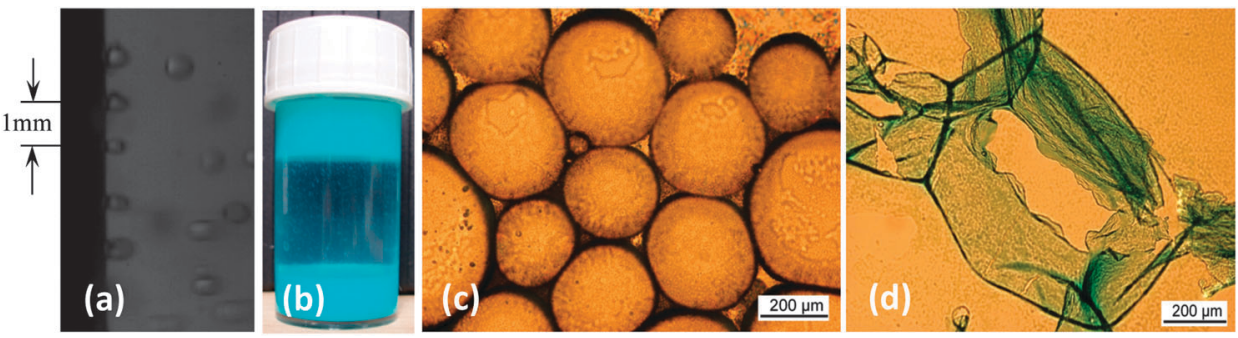

Fig. 2 Oil-in-water emulsions were produced by a rotating membrane emulsification method. (a) Droplet formation process through the membrane rotating at $300 \mathrm{rpm}$ (recorded by a high speed camera at 3000 frames per s); (b) emulsion sample (top layer: oil droplets; middle layer: aqueous phase and bottom layer: excess particles); (c) the formed oil droplets in a range of 200-300 $\mu \mathrm{m}$; (d) the MOF particle film formed on the droplet surface.

of the formed oil-in-water droplets were in a range of $\sim 200-300 \mu \mathrm{m}$ in diameter (Fig. 2c), which was proportional to the membrane pore size of $80 \times 80 \mu \mathrm{m}$. This result was consistent with a previous study in which affinitive surfactants were used as stabilisers. ${ }^{9}$

Fig. $2 \mathrm{~d}$ shows the remains of the blue MOF particle film after the aqueous phase was evaporated, followed by the oil droplets being broken. Neglecting the apertures, the monolayer particle film had a good continuity; it played an exceptional role in stabilisation of the droplets in the continuous phase. The film thickness and the number of MOF particles in the film depend on the MOF particle size (or volume) that also affects the nanopore volume of the film. For example, at the given droplet diameter, a decrease of MOF particle size from 300 to $200 \mathrm{~nm}$ will theoretically increase the particle number by 2.25 fold, and decrease the nanopore volume by one third. These could influence the microdroplets as the potential vehicle to deliver interesting species, e.g. antimicrobials.

The impact of particle adsorption at the interface on the dynamic interfacial tension was investigated by pending an aqueous droplet in the oil (Fig. 3). With the adsorption of particles, the interfacial tension gradually reduced from $\sim 12-14$ to $\sim 6-7 \mathrm{mN} \mathrm{m}^{-1}$ and levelled at this value afterwards. The equilibrium time was governed by the wetting kinetics of the MOF particles.

In summary, the nanoporous Cu-BTC MOF nanoparticles have exhibited an exceptional function as a sole stabiliser to stabilise both water-in-oil and oil-in-water emulsions without further surface modification. The MOF nanoparticles were adsorbed at the oil-water interface to form a continuous thin film preventing the droplets from coalescing. Dynamic adsorption of particles at the droplet surface determines the droplet size and distribution. Both water-in-oil and oil-in-water emulsions formed in this study have excellent stabilities. This unique property is attributed to the hybrid chemical nature of

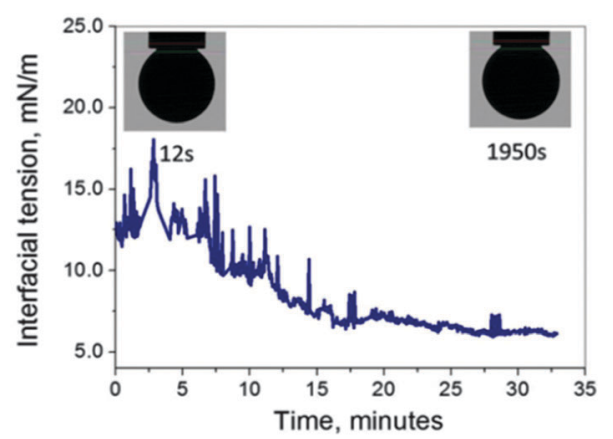

Fig. 3 Interfacial tension profile of an aqueous droplet in the oil phase measured by the pending drop method. the MOF frameworks, leading to MOFs which are advantageous over other surface active particles potentially for applications in controlled drug delivery, catalysis and microreaction. Nanoporous MOF particle stabilising emulsions provides a new approach to construct multifunctional micro-structures, and is worthy of further exploration.

\section{Notes and references}

1 (a) M. Kargar, K. Fayazmanesh, M. Alavi, F. Spyropoulos and I. T. Norton, J. Colloid Interface Sci., 2012, 366, 209; (b) J. Frelichowska, M.-A. Bolzinger, J.-P. Valour, H. Mouaziz, J. Pelletier and Y. Chevalier, Int. J. Pharm., 2009, 368, 7; (c) B. Isabelle and T. Philippe, US Pat., 5637291, 1997; (d) S. Laurier and S. Elaine, Finely Dispersed Particles, CRC Press, 2005, 79; (e) S. U. Pickering, J. Chem. Soc. Trans., 1907, 91, 2001; $(f)$ A. D. Dinsmore, M. F. Hsu, M. G. Nikolaides, M. Marquez, A. R. Bausch and D. A. Weitz, Science, 2002, 298, 1006; $(g)$ S. Crossley, J. Faria, M. Shen and D. E. Resasco, Science, 2010, 327, 68.

2 (a) T. S. Horozov and B. P. Binks, Angew. Chem., Int. Ed., 2006, 45, 773; (b) R. Aveyard, B. P. Binks and J. H. Clint, Adv. Colloid Interface Sci., 2003, 100-102, 503.

3 S. Levine, B. D. Bowen and S. J. Partridge, Colloids Surf., 1989, 38, 325. 4 (a) B. P. Binks, J. Philip and J. A. Rodrigues, Langmuir, 2005, 21, 3296; (b) P. Wongkongkatep, K. Manopwisedjaroen, P. Tiposoth, S. Archakunakorn, T. Pongtharangkul, M. Suphantharika, K. Honda, I. Hamachi and J. Wongkongkatep, Langmuir, 2012, 28, 5729; (c) J. W. J. de Folter, M. W. M. van Ruijven and K. P. Velikov, Soft Matter, 2012, 8, 6807; (d) K. Larson-Smith and D. C. Pozzo, Langmuir, 2012, 28, 11725; (e) M. Williams, S. P. Armes and D. W. York, Langmuir, 2011, 28, 1142; $(f)$ J. Zhou, X. Qiao, B. P. Binks, K. Sun, M. Bai, Y. Li and Y. Liu, Langmuir, 2011, 27, 3308; ( $g$ ) E. A. Zaragoza-Contreras, C. A. HernándezEscobar, A. Navarrete-Fontes and S. G. Flores-Gallardo, Micron, 2011, 42, 263; $(h)$ Q. Yuan, O. J. Cayre, S. Fujii, S. P. Armes, R. A. Williams and S. Biggs, Langmuir, 2010, 26, 18408.

5 (a) H. Furukawa, N. Ko, Y. B. Go, N. Aratani, S. B. Choi, E. Choi, A. Ö. Yazaydin, R. Q. Snurr, M. O'Keeffe, J. Kim and O. M. Yaghi, Science, 2010, 329, 424; (b) A. M. Shultz, A. A. Sarjeant, O. K. Farha, J. T. Hupp and S. T. Nguyen, J. Am. Chem. Soc., 2011, 133, 13252; (c) K. Sumida, D. L. Rogow, J. A. Mason, T. M. McDonald, E. D. Bloch, Z. R. Herm, T.-H. Bae and J. R. Long, Chem. Rev., 2012, 112, 724; (d) L. Ma, J. M. Falkowski, C. Abney and W. Lin, Nat. Chem., 2010, 2, 838; (e) S. Bureekaew, S. Horike, M. Higuchi, M. Mizuno, T. Kawamura, D. Tanaka, N. Yanai and S. Kitagawa, Nat. Mater., 2009, 8, 831; $(f)$ B. Xiao, P. S. Wheatley, X. Zhao, A. J. Fletcher, S. Fox, A. G. Rossi, I. L. Megson, S. Bordiga, L. Regli, K. M. Thomas and R. E. Morris, J. Am. Chem. Soc., 2007, 129, 1203; (g) A. C. McKinlay, R. E. Morris, P. Horcajada, G. Férey, R. Gref, P. Couvreur and C. Serre, Angew. Chem., Int. Ed., 2010, 49, 6260; (h) P. D. C. Dietzel, B. Panella, M. Hirscher, R. Blom and H. Fjellvåg, Chem. Commun., 2006, 959; (i) P. D. C. Dietzel, Y. Morita, R. Blom and H. Fjellvåg, Angew. Chem., Int. Ed., 2005, 44, 6354.

6 S. S.-Y. Chui, S. M.-F. Lo, J. P. H. Charmant, A. G. Orpen and D. Williams, Science, 1999, 283, 1148.

7 M. J. Ingleson, R. Heck, J. A. Gould and M. J. Rosseinsky, Inorg. Chem., 2009, 48, 9986.

8 Q. Yuan, O. J. Cayre, M. Manga, R. A. Williams and S. Biggs, Soft Matter, 2010, 6, 1580.

9 Q. Yuan, N. Aryanti, R. Hou and R. A. Williams, Particuology, 2009, 7, 114. 